\title{
Robust Error Concealment Algorithm for MPEG-4 with the Aids of Fuzzy Theory
}

\author{
Pei-Jun Lee ${ }^{1}$, Liang-Gee Chen ${ }^{1}$, Wen-June Wang ${ }^{2}$, and Mei-Juan Chen ${ }^{3}$ \\ 'Department of Electrical Eng., National Taiwan University, \\ ${ }^{2}$ Department of Electrical Eng., National Central University, \\ ${ }^{3}$ Department of Electrical Eng., National Dong-Hwa University,
}

\begin{abstract}
A new error concealment algorithm based on fuzzy set theory is proposed to repair damaged portions of the shape information. By the experiment results, the proposed algorithm has better performance than previous works especially on the case that the damaged portion includes the edge of object.
\end{abstract}

\section{Introduction}

In the last few years, more and more sophisticated digital video communication services and devices emerged in the market. Traditional video standard assumes that the compressed video bit-streams transmit in error-free medium. However, it is well known that the compressed video bit-streams are very sensitive to channel errors. MPEG-4 standard has not such limitation and it supports an object-based representation by allowing the coding of the shape information of arbitrarily shaped video objects along with the objects' texture and motion information. Therefore, the correctness of shape information is much more important than the other information.

When variable-length code (VLC) technique is used in the shape coding, variable-length characteristic inherently will makes VLC sensitive to noise errors. Even a single error in a video bit-stream may have a large influence on the video quality. Although previous works have shown that utilizing synchronization codeword or packet [1] can avoid error propagation. However, the efficient concealment algorithms [2] of the shape information in the MPEG-4 are still important in decoding work since they provide an acceptable approximation of the original data.

\section{The algorithms}

The spatial interpolation is the simplest error concealment method $[3,4]$ under any cases such as I-frame, B-frame and $\mathrm{P}$-frame. But there may be a problem happening when the interpolation is used in the alpha plane with boundary blocks. In other words, after interpolation the boundary blocks will produce a cloudy region, therefore the exact location of boundary is difficult to be identified. This paper uses a method based on fuzzy set theory to solve this problem. In each row of a boundary block, we set a fuzzy set on the vague boundary interval. Then by the center of gravity defuzzification technique, a value in the corresponding row is obtained to determine the boundary in the row. We take care every row of the boundary block by the same manner such that the boundary will be found. The procedure of our algorithm is summarized in the following flow chart.

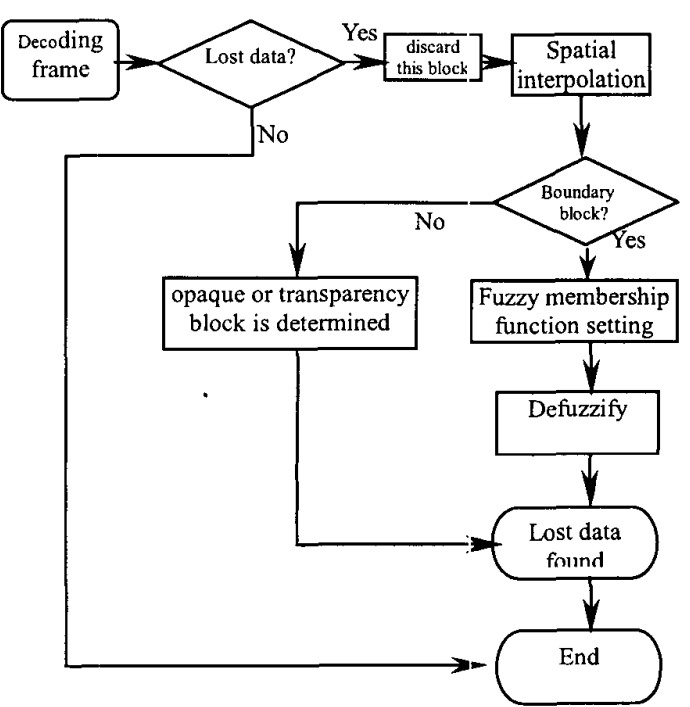

Fig. 1 The system diagram of the proposed algorithm

In Fig. 1, the formula for fuzzy membership is set

$$
f_{i}\left(X_{i j}\right)= \begin{cases}\frac{T_{1}-X_{i j}}{T_{1}-127}, & \text { for } T_{1} \leq X_{i j} \leq 127 \\ \frac{X_{i j}-T_{2}}{127-T_{2}}, & \text { for } 127 \leq X_{i j} \leq T_{2}\end{cases}
$$

where $T_{1}$ and $T_{2}$ are some values from opaque and transparency, respectively, $X_{i j}$ is the gray value of the pixel with coordinate $(i, j)$ in the block. Moreover, the formula for defuzzification is $\sum_{j} f_{i}\left(X_{i j}\right) X_{i j}$ where 
$\hat{X}_{i j}$ is the defuzzified value.

\section{Results}

Experimental results will demonstrate that the proposed algorithm improves significantly in terms of objective (shown in Table 1) and subjective (shown in Fig. 2) measurements compared with other methods. We can conclude that the proposed algorithm is more efficient and robust for recovering the lost data in the shape information.

Table.1 Average PSNR and Pixel error comparisons for various schemes

\begin{tabular}{|c|c|c|c|c|c|c|}
\hline Sequence & \multicolumn{6}{|c|}{ Bream } \\
\hline \multirow[b]{2}{*}{ frames $/ \mathrm{sec}$} & \multicolumn{2}{|c|}{ S I } & \multicolumn{2}{|c|}{ AMV } & \multicolumn{2}{|c|}{ Proposed } \\
\hline & PSNR & PSE & PSNR & PSE & PSNR & PSE \\
\hline 30 & 16.5 & 167.66 & 17.74 & 145.33 & 18.56 & 105.81 \\
\hline 15 & 16.45 & 184.79 & 17.54 & 171.34 & 17.94 & 130.3 \\
\hline 10 & 15.87 & 208.2 & 16.87 & 190.13 & 17.12 & 156.53 \\
\hline Sequence & \multicolumn{6}{|c|}{ Children } \\
\hline \multirow[b]{2}{*}{ frames $/ \mathrm{sec}$} & \multicolumn{2}{|c|}{ S I } & \multicolumn{2}{|c|}{ AMV } & \multicolumn{2}{|c|}{ Proposed } \\
\hline & PSNR & PSE & PSNR & PSE & PSNR & PSE \\
\hline 30 & 14.48 & 226.92 & 15.09 & 215.98 & 16.65 & 136.67 \\
\hline 15 & 12.98 & 250.72 & 13.11 & 209.28 & 15.42 & 160.33 \\
\hline 10 & 12.61 & 274.02 & 12.49 & 329.87 & 14.88 & 185.08 \\
\hline
\end{tabular}

\section{Reference}

[1] Y.S Lee, W.S Chang, H.H Ho and C.Y Lee, "Construct of error resilient synchronization codeword for variable-length code in image transmission," IEEE International Conference on Image Processing, 2000.

[2] S. Shirani, B. Erol, and F. Kossentini, "A concealment method for shape information in MPEG-4 coded video sequences," IEEE Tran. on Multimedia. Vol.2, No.3, September 2000.

[3] P.J. Lee and M.J. Chen, "Robust recovery algorithm for video coding," IEEE Trans. on Consumer Electronic, Vol.45, No.3, pp.851-859, August 1999.

[4] M.J. Chen, L.G. Chen, and R.M. Weng, "Error concealment of lost motion estimation vectors with overlapped motion compensation," IEEE Trans. on Circuits and Systems for Video Technology, Vol.7, No.3,pp.560-563,June 1997.

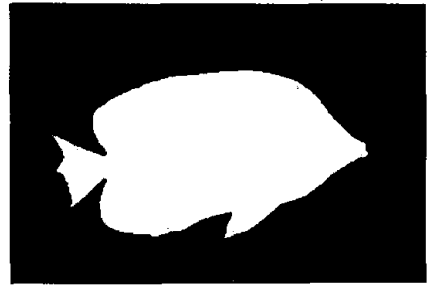

(1)

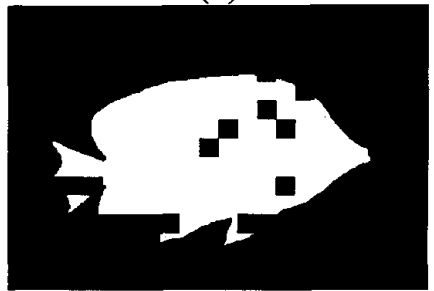

(2)

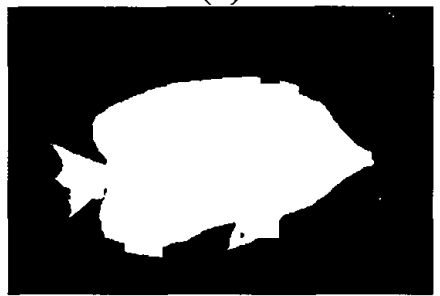

(3)

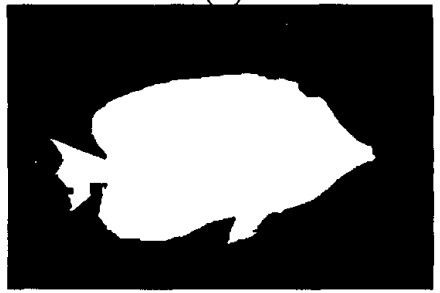

(4)

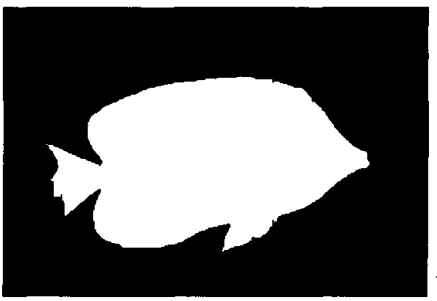

(5)

Fig.2 Reconstructed pictures for Table Bream shape Sequence. (1) The original picture, (2) The damaged picture. (3) The recovered picture by above motion vector, (4) The recovered picture by spatial interpolation threshold is 127, (5) The recovered picture by the proposed method. 